\title{
DINÂMICA ESPAÇO-TEMPORAL DA TEMPERATURA DE SUPERFÍCIE, EXTRAÍDA DO TM/LANDSAT, NA BACIA DO CORUMBATAÍ, SP
}

Article $\cdot$ April 2016

DOI: 10.17224/EnergAgric.2016v31n2p169-177

CITATIONS

0

5 authors, including:

Rodrigo Almeida Muniz

Universidade Federal do Sul e Sudeste do P.

21 PUBLICATIONS 49 CITATIONS

SEE PROFILE
Lucieta Guerreiro Martorano

Brazilian Agricultural Research Corporation...

112 PUBLICATIONS 49 CITATIONS

SEE PROFILE 


\title{
DINÂMICA ESPAÇO-TEMPORAL DA TEMPERATURA DE SUPERFÍCIE, DA BACIA DO RIO CORUMBATAÍ, SP UTILIZANDO IMAGENS DO SENSOR TM/LANDSAT
}

\author{
Rodrigo Almeida Muniz, Carlos Alberto Vettorazzi, Lucieta Guerreiro Martorano, Carlos \\ Tadeu Santos Dias \& Leila Sheila Silva Lisboa
}

\begin{abstract}
RESUMO: Neste estudo foram empregados sistemas de informações geográficas e técnicas de sensoriamento remoto para investigar o efeito do uso e cobertura do solo sobre a temperatura da superfície do solo (TSS) nos anos de 1985, 1990, 1995, 1999, 2002 e 2011, na bacia do Rio Corumbataí (BRC), SP. O padrão espacial da TSS foi derivado de imagens do satélite Landsat, por meio da banda termal do sensor TM. Estudou-se a relação entre os índices NDVI, NDBI, MI e da altitude com a TSS. A TSS da BRC foi crescente até o ano de 1999 e reduziu até o ano de 2011 . O padrão espacial da TSS foi influenciado pelo uso e cobertura do solo. Houve correlação negativa da TSS com o NDVI e MI e positiva com o NDBI. Áreas de pastagem e áreas de cana-de-açúcar colhidas podem atingir TSS superiores às áreas urbanas, dependendo das condições de umidade do ambiente. Conclui-se que os dados do TM/Landsat associados aos dados coletados em campo, apresentam potencial para identificação de padrões térmico-hídricos em estudos de bacias hidrográficas.
\end{abstract}

PALAVRAS-CHAVES: Sensoriamento remoto, Índices de vegetação, Uso e cobertura do solo.

\section{SPATIAL AND TEMPORAL DYNAMICS OF LAND SURFACE TEMPERATURE FROM TM/LANDSAT, IN CORUMBATAI BASIN, SP}

\begin{abstract}
In this study, Geographical Information System and Remote Sensing techniques were employed in order to investigate the effect of land use/land cover on land surface temperature (LST) for 1985, 1990, 1995, 1999, 2002, and 2011, in the Corumbatai River basin (BRC) located in Sao Paulo. The LST spatial pattern was derived from the thermal band image of the TM/Landsat sensor. It was studied the relationship between NDVI, NDBI, MI, altitude, and LST. The LST of the BRC increased by 1999 and decreased by 2011. Land use/land cover influences LST spatial pattern. There was a negative correlation between LST and both NDVI and MI, and a positive correlation with NDBI. Pasture and harvested sugarcane areas can present higher LST than build-up land, depending on the environment moisture conditions. Therefore, we conclude that the association between the data from the TM/Landsat and the field data has potential to identify thermal hydric patterns in basins studies.
\end{abstract}

KEYWORDS: Remote sensing; Vegetation Index; Land use/land cover.

\section{INTRODUÇÃO}

O padrão de distribuição térmica, expresso pela temperatura da superfície do solo (TSS), é um dos principais efeitos relacionados à antropização, principalmente nos grandes centros urbanos. A TSS pode ser influenciada pelo tipo de uso e cobertura do solo, geralmente com maiores temperaturas nos centros urbanos e menores nas áreas rurais em seu entorno. Entretanto, dependendo do tipo de uso e da forma com que o solo é utilizado, este padrão, pode ser igualado ou mesmo invertido. Dependendo da intensidade da mudança nos padrões de distribuição térmica, estes podem alterar a temperatura regional e, consequentemente, impactar as interações no sistema solo-corpos hídricos- planta-atmosfera (LIU et al., 2012; ORHAN, EKERCIN E DADASER-CELIK, 2014).

Mudanças na TSS podem ser influenciadas pela alteração do balanço de energia. Em superfícies vegetadas com alto índice de área foliar, a maior fração do saldo de radiação (SR) é alocada para a evapotranspiração das plantas, consequentemente, reduzindo as frações destinadas ao aquecimento do ar e do solo. Da mesma forma, superfícies sem cobertura vegetal alocam maiores frações do SR para o aquecimento do ar e, principalmente, do solo. Quando a temperatura do solo é maior que a temperatura do ar, a energia armazenada no solo é transferida por condução às camadas de ar próximas à superfície. $\mathrm{O}$ aquecimento dessas camadas gera um gradiente de pressão e, por convecção, a 
energia é transportada às camadas superiores de ar (ZHANG et al., 2014).

Diferentes materiais da superfície, como edificações, asfalto e concreto, têm alta retenção de calor e baixa emissividade, resultando em um aumento do fluxo de calor sensível e menor fluxo de calor latente, quando comparados as superfícies vegetadas (LIU et al., 2012; ZHAN et al., 2012).

A temperatura da superfície pode ser obtida através de imagens de sensores orbitais como os do satélite Landsat. Qin, Karnielli e Berliner (2001) desenvolveram um algoritmo, baseado na equação de transferência térmica, para estimar a TSS a partir de dados obtidos da banda 6 do sensor TM/LANDSAT. O algoritmo requer três parâmetros atmosféricos: transmitância, emissividade e temperatura atmosférica média.

Mudanças nas características da superfície podem ser monitoradas por meio de índices obtidos por sensoriamento remoto. Características de interceptação da radiação do dossel das plantas, que estão diretamente ligadas à fotossíntese, resistência estomática e evapotranspiração podem ser inferidas da medida da radiação solar refletida pela banda do infravermelho próximo e do vermelho do satélite LANDSAT. Tais características podem ser expressas por meio do NDVI (Normalized Difference Vegetation Index (CHEN et al., 2014; ORHAN, EKERCIN E DADASER-CELIK, 2014).

Informações de áreas urbanas e áreas com baixa cobertura vegetal podem ser extraídas pelo índice NDBI (Normalized Difference Built-up Land Index) que se baseia no fato de áreas construídas e solo exposto terem maior refletância no infravermelho próximo do que no infravermelho médio, destacando principalmente áreas urbanas e solo exposto (ZHANG, ODEH E HAN, 2009).

O índice MI (Moisture Index) não fornece a estimativa da quantidade de umidade do solo, mas é sensível a variedades de tipos de superfícies úmidas, relativas a outras propriedades dentro de uma determinada cena, além de ser sensível a uma grande variedade de tipos de cobertura e robusto o suficiente para ser independente de medidas in situ (DUPIGNY-GIROUX \& LEWIS, 1999).

O objetivo deste trabalho foi verificar se o tipo de uso e cobertura do solo influencia na temperatura de superfície por meio da análise integrada da altitude, e dos índices de vegetação: NDVI, NDBI e MI, nos anos de 1985, 1990, 1995, 1999, 2002 e 2011 da Bacia do Rio Corumbataí, SP.

\section{MATERIAL E MÉTODOS}

\section{1 Área de estudo}

A bacia do Rio Corumbataí (BRC) está localizada entre os paralelos $22^{\circ} 04^{\prime} 46^{\prime}$ ' S e $22^{\circ} 41^{\prime} 28^{\prime \prime} \mathrm{S}$ e os meridianos $47^{\circ}$ $27^{\prime} 40^{\prime}$ W e $47^{\circ} 55^{\prime} 03$ " W. O perímetro é de aproximadamente $301 \mathrm{~km}$, com $63 \mathrm{~km}$ de extensão na direção N-S e 46 km na direção L-O. Possui uma área de $1.710 \mathrm{~km}^{2}$, abrangendo os municípios de Corumbataí, Ipeúna, Rio Claro e Santa Gertrudes e parte dos municípios de Analândia, Charqueada, Itirapina e Piracicaba. A BRC integra o sistema Tietê-Paraná, sendo uma sub-bacia da margem direita do rio Piracicaba.

A altitude da bacia está na faixa de 470 a 1.058 m, da foz à nascente. O clima da região, conforme a classificação de Köppen, é do tipo Cwa, subtropical seco no inverno e chuvoso no verão e temperatura média do mês mais quente superior a $22^{\circ} \mathrm{C}$.

As temperaturas mais elevadas ocorrem nos meses de dezembro a março e as mais baixas nos meses de junho e julho, sendo a temperatura média próxima a $17^{\circ} \mathrm{C}$. $\mathrm{O}$ regime de chuva é típico de clima tropical, com duas estações bem definidas, um período seco de março a setembro e um período chuvoso de outubro a fevereiro, período que responde por mais de $80 \%$ da precipitação anual, sendo esta de $1.390 \mathrm{~mm}$. A insolação média é de 2.420 horas e os ventos são moderados durante o ano, com direção predominante de E-SE (TROPPMAIR, 1974).

\subsection{Base de dados}

Foram utilizados dados de temperatura do ar obtidos em duas estações meteorológicas: $\left(1^{\circ}\right)$ Estação Meteorológica de Rio Claro, SP (CEAPLA/IGCE e Prefeitura Municipal) Esta estação é do tipo convencional completa, situada nas coordenadas geográficas $22^{\circ} 23^{\prime} \mathrm{S}$ e $47^{\circ} 32^{\prime} \mathrm{W}$ e altitude de 626,5 m. A estação iniciou a coleta de dados em 1994, com quatro observações diárias a 1,5 m de altura às 7:00, 9:00, 15:00 e 21:00 h e a partir de 2010 em intervalos de 10 minutos; $\left(2^{\circ}\right)$ Estação meteorológica da ESALQ/USP Localizada nas coordenadas geográficas $22^{\circ} 42^{\prime} \mathrm{S}$, 47o 38' W e altitude de $546 \mathrm{~m}$, na cidade de Piracicaba, SP, com coleta de temperatura do ar desde 1917 a 1,5 m de altura.

Os mapas de uso e cobertura do solo dos anos de 1999 e 2002 foram obtidos, respectivamente, de Valente e Vettorazzi (2002 e 2008), a partir da classificação supervisionada de imagens do sensor TM/Landsat datadas de 02/09/1999 e 17/08/2002. O modelo numérico do terreno utilizado foi o SRTM (2010) com resolução espacial de 30 x $30 \mathrm{~m}$, interpolado por Valeriano et al. (2006).

\subsection{Pré-processamento e classificação digital de imagem}

Foram utilizadas as imagens do sensor TM dos satélites Landsat-5 e 7, orbita 220 e pontos 75 e 76 , das seguintes datas: 10/08/1985, 08/08/1990, 07/09/1995 e 19/09/2011 As imagens foram corrigidas geometricamente por meio da imagem Geocover do satélite Landsat-7, sensor ETM+ e, retificadas para o sistema de coordenadas UTM, zona 23S. O erro quadrado médio máximo aceitável adotado foi de 0,5 pixel.

As imagens foram classificadas em seis classes de uso: cana-de-açúcar, pastagem, remanescentes florestais, plantios florestais, fruticultura, áreas urbanas e outros. A classe "outros" incluiu áreas de mineração, baixadas alagadas e pequenos corpos hídricos. Foi empregado o método de classificação supervisionada, utilizando o algorítimo de máxima verossimilhança (MAXVER) do programa ENVI 4.3. O produto obtido foi editado para a 
correção de erros de classificação do algoritmo, com base em pesquisas de campo, imagens orbitais de épocas diferentes e conhecimento da área pelo intérprete. A exatidão global da classificação de 2011 foi de 92\%. Considerou-se que as outras classificações possuem a mesma exatidão que a de 2011.

\subsection{Estimativa da TSS}

A correção atmosférica antecedeu a estimação da TSS, por meio da conversão dos níveis de cinza à radiância espectral, utilizando os coeficientes de calibração radiométrica, obtidos no cabeçalho das imagens (CHANDER, MARKAHAM E HELDER., 2009). As imagens de radiância espectral foram convertidas à temperatura de brilho do satélite (TB), seguindo os pressupostos de Chander e Markham (2003). A partir da TB, a TSS foi obtida pelo algoritmo mono-window, proposto por Qin, Karnieli e Berliner (2001). Para a obtenção da TSS, foi considerada a emissividade como função do NDVI, de acordo com a equação proposta por Van De Griend e Owe (1993). O desempenho da TSS foi obtido comparando-se os valores estimados de TSS com os valores observados de temperatura do ar, coletados nas estações meteorológicas.

\section{5 Índices de sensoriamento remoto}

Foram utilizados os índices NDVI, NDBI e MI. Estes foram calculados utilizando as seguintes bandas espectrais do satélite TM/LANDSAT: banda 1 de 0,45 a $0,52 \mu \mathrm{m}$; banda 3 de 0,63 a $0,69 \mu \mathrm{m}$; banda 4 de 0,76 a $0,9 \mu \mathrm{m}$ e banda 5 de 1,55 a $1,75 \mu \mathrm{m}$, de acordo com as seguintes equações:

$$
\begin{aligned}
& \text { NDVI }=\frac{(\text { Banda } 4-\text { Banda } 3)}{(\text { Banda } 4+\text { Banda } 3)} \\
& \text { NDBI }=\frac{(\text { Banda } 5-\text { Banda } 4)}{(\text { Banda } 5+\text { Banda } 4)}
\end{aligned}
$$

$\mathrm{MSI}=\frac{\text { Banda } 4}{\text { Banda } 1}$

\subsection{ANÁLISES ESTATÍSTICAS}

Para as análises estatísticas, foram extraídos 5.000 pontos aleatórios dos planos de informação (PI): uso e cobertura do solo, LST, NDVI, NDBI, MI e MNT, no software ArcGIS 9.3. Os pontos foram extraídos na forma de tabelas onde procedeu-se a análise exploratória dos dados. A TSS foi submetida ao teste $\mathrm{T}$ de médias pareadas, a $5 \%$ de probabilidade. A relação entre as variáveis NDVI, NDBI, MI, MNT e TSS, foi obtida por meio da análise de componentes principais. Todas as análises foram feitas utilizando os pacotes stats, agricolae e ggbiplot implementados no software R (R CORE TEAM, 2013).

\section{RESULTADOS E DISCUSSÃO}

\subsection{Condições meteorológicas na ocasião da imagem}

De acordo com a Figura 1, observa-se que as imagens de 1985, 1995 e 1999 foram precedidas de um período de estiagem superior a 30 dias. Nos anos de 1990, 2002 e 2011, o período de estiagem foi menor, respectivamente, com 13, 10 e 19 dias, com precipitações, respectivamente, de 29, 71,6 e $10 \mathrm{~mm}$. Nos anos avaliados, de 1985 a 2011, a temperatura média foi, respectivamente, de $21,19,20,23,21^{\circ} \mathrm{C}$, a umidade relativa média de $51,68,47,50,50$ e $45 \%$, a radiação solar global média de 435, 344, 452, 422, 415 e 557 cal $\mathrm{cm} \mathrm{dia}^{-1}$, a velocidade do vento média foi $8,9,6,2,6$, e $7 \mathrm{~m} \mathrm{~s}^{-1}$, e a evaporação média de $2,3,2$ e $4 \mathrm{~mm} \mathrm{dia}^{-1}$. Não houve dados disponíveis para os dias 17/08/02 e 19/09/2011 referentes à evaporação média diária (Figura 1).
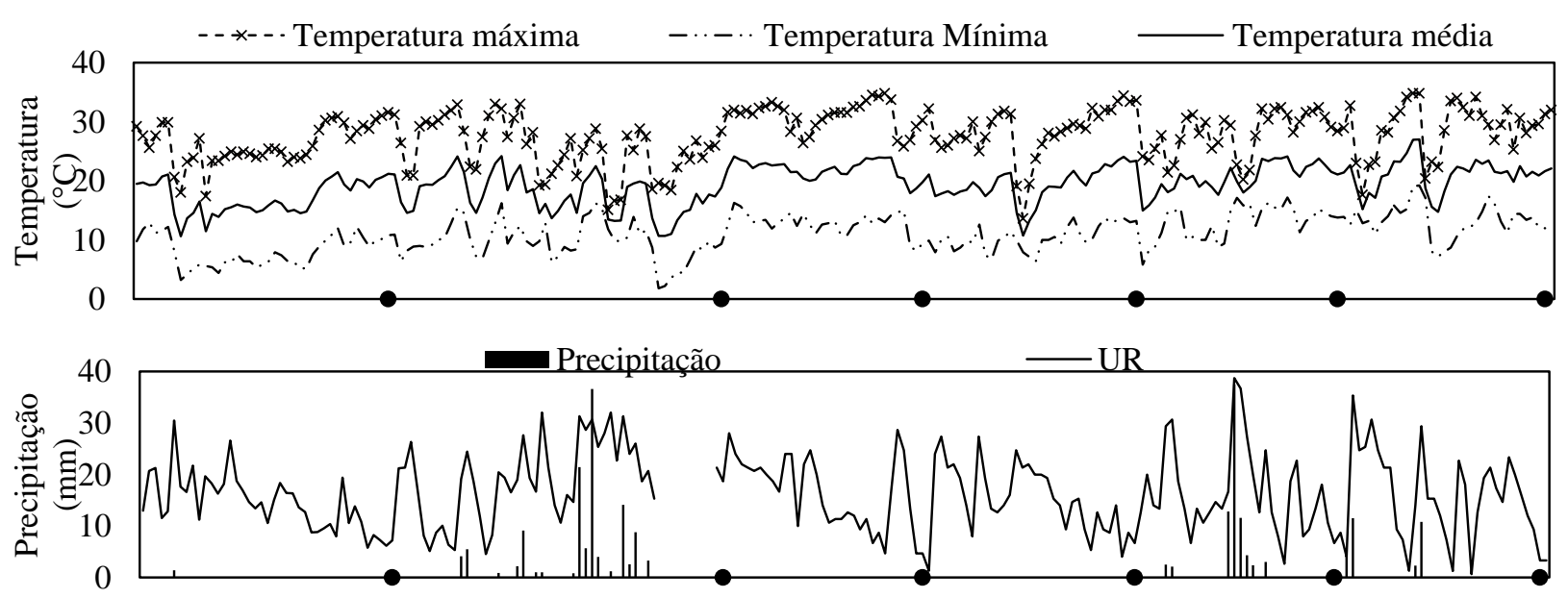


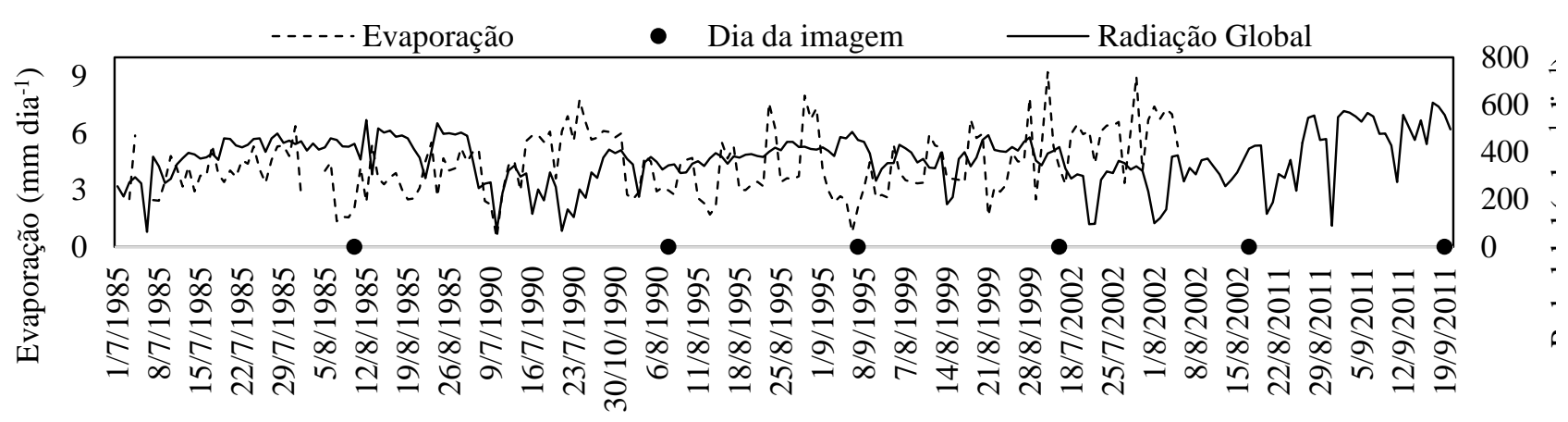

Figura 1 - Condições meteorológicas predominantes na bacia do Rio Corumbataí no período de 30 dias anteriores à passagem do satélite.

\subsection{Validação da TSS}

Há incertezas associadas a estimativa da TSS que podem estar relacionadas ao sincronismo da passagem do satélite com a medição da temperatura pela estação, porém, para a validação da TSS utilizou-se dados medidos próximos a passagem do satélite. O coeficiente de correlação de Pearson foi de 0,92 . O coeficiente de determinação $\left(R^{2}\right)$ da regressão linear, com intercepto igual a zero, foi de 0,7 (Figura 2). A TSS superestimou a temperatura da estação, o que já era esperado, pois, segundo Grodzki et al. (1996), dependendo da cobertura da superfície do solo pode haver diferenças de até $10^{\circ}$ entre a temperatura do ar, a $1,5 \mathrm{~m} \mathrm{e}$, a temperatura na superfície do solo. Tal diferença é devido ao impacto de possíveis efeitos no tranporte de massa e energia pelas microadvecções, sendo influenciada pela mistura atmosférica, velocidade média do vento e as propriedades térmicas dos materiais em superfície (STOLL E BRAZEL, 1992).

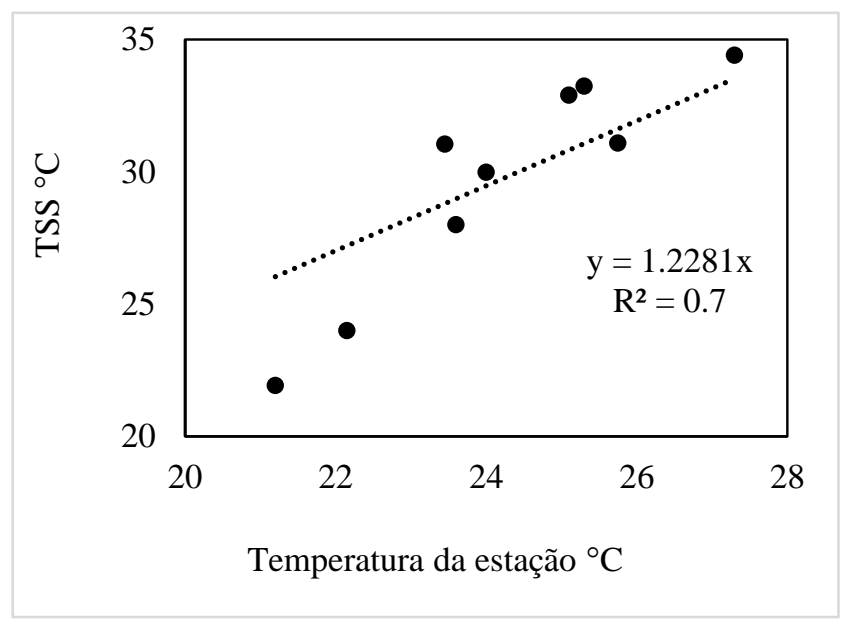

Figura 2 - Temperatura do ar medida na estação e temperatura da superfície obtida por sensoriamento remoto.

\subsection{Dinâmica do uso e cobertura da terra}

O principal uso da BRC foi cana-de-açúcar e pastagem (Figura 3). De 1985 a 2011, a pastagem reduziu 21\%, os remanescentes florestais aumentaram $7 \%$, seguidos por cana-de-açúcar, cultivos florestais, fruticultura e áreas urbanas, com incremento, respectivamente, de 5, 3, 3 e $2 \%$.
$\mathrm{Na}$ porção norte da BRC predominou a pastagem e na porção sul a cana-de-açúcar. A principal mudança de uso do solo ocorreu na porção sul da bacia, onde prevaleceu a conversão pastagem à cana-de-açúcar. Próximo à nascente as principais mudanças foram a expansão de plantios florestais e fruticultura. 


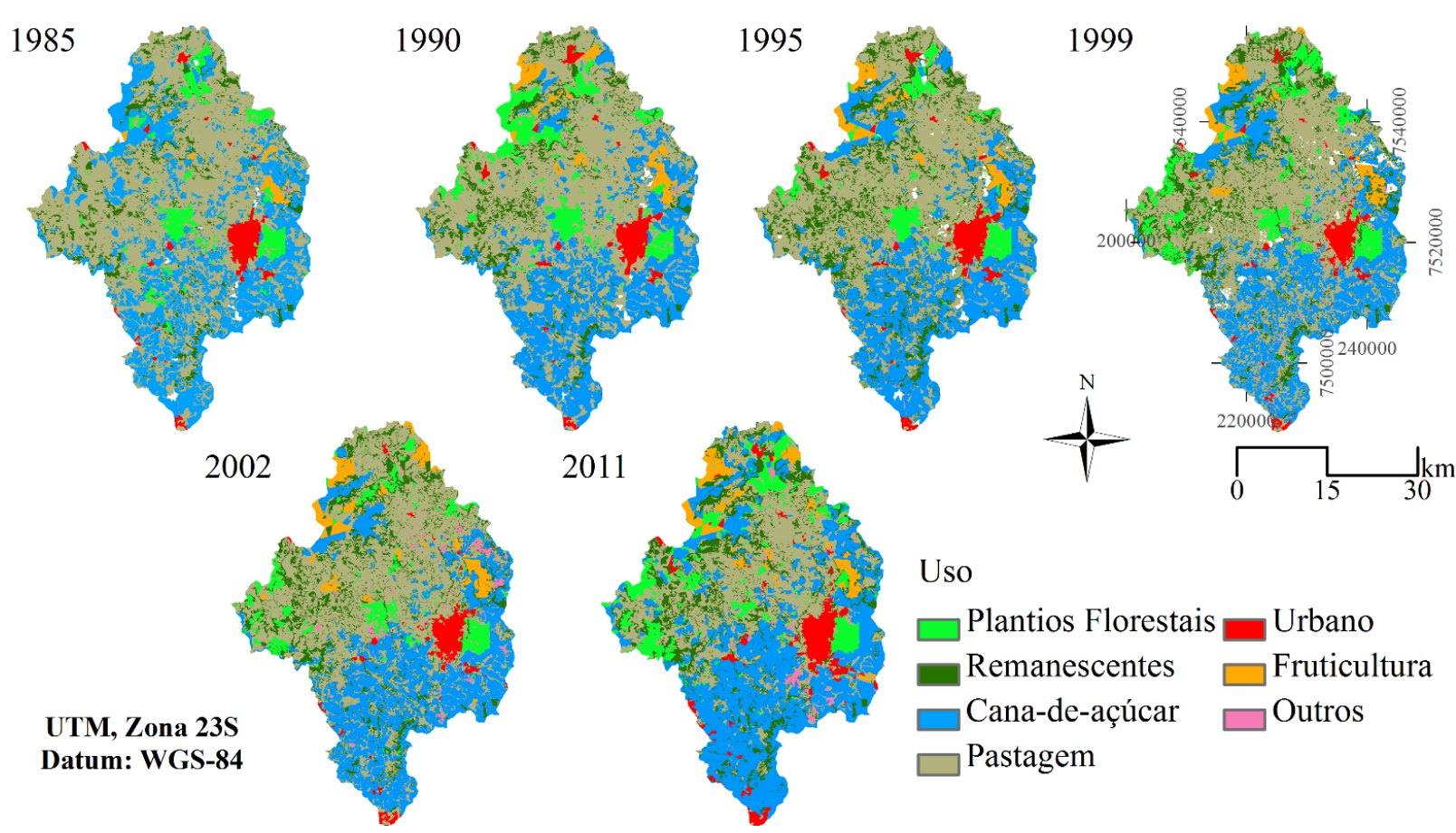

Figura 3 - Dinâmica espaço-temporal de uso e cobertura da terra na Bacia do Rio Corumbataí, SP.

\subsection{Efeitos do uso e cobertura do solo sobre a TSS}

A TSS média da BRC foi submetida ao teste T de students $(\mathrm{P}<0,05)$, apresentando diferença significativa entre todos os anos. O menor valor observado foi em 1985 de $20^{\circ} \mathrm{C}$ e os maiores valores foram 31 e $32^{\circ} \mathrm{C}$, respectivamente, em
2002 e 1999. A temperatura média local (TML) no período de estudo, agosto e setembro, com base nos dados das estações meteorológicas, foi de $22^{\circ} \mathrm{C}$. Tomando a TML como referência, calculou-se a diferença em relação a TSS média de cada classe de uso (Tabela 1).

Tabela 1 - Diferença entre a temperatura média local dos meses de agosto e setembro (22 $\mathrm{C}$ ) e a temperatura de cada classe de uso do solo

\begin{tabular}{lllllll}
\hline Uso e cobertura & 1985 & 1990 & 1995 & 1999 & 2002 & 2011 \\
\hline Plantios florestais & 5,0 & 1,1 & $-0,5$ & $-7,9$ & $-7,3$ & $-5,1$ \\
Remanescentes & 4,2 & 0,4 & $-2,2$ & $-8,6$ & $-7,3$ & $-5,3$ \\
Cana-de-açúcar & 2,1 & $-0,9$ & $-4,3$ & $-11,4$ & $-9,4$ & $-9,6$ \\
Pastagem & 1,9 & $-1,6$ & $-4,6$ & $-10,9$ & $-9,2$ & $-8,8$ \\
Áreas urbanas & 0,8 & $-2,1$ & $-4,7$ & $-11,1$ & $-10,4$ & $-9,8$ \\
Fruticultura & 2,2 & $-2,7$ & $-4,8$ & $-12,7$ & $-8,9$ & $-9,5$ \\
Outras & 1,1 & $-2,0$ & $-4,4$ & $-10,2$ & $-9,2$ & $-8,3$ \\
\hline
\end{tabular}

Observa-se na Tabela 1 que a TSS em 1985 de todas as classes de uso foi inferior à TML. Em 1990, apenas os plantios florestais e mata nativa foram inferiores à TML e a partir de 1995, a TSS foi superior a TMR em todas as classes. A maior diferença foi no ano de 1999, na fruticultura, com um valor de 12,73. Tais resultados mostram que as classes remanescentes florestais e plantios florestais são mais resilientes, quanto ao aumento da temperatura, e áreas de agricultura, pastagem e áreas urbanas estão mais sujeitas às oscilações da temperatura, principalmente nos anos de temperatura mais elevada.
Áreas com intensa cobertura vegetal foram reduzidas causando o aumento da temperatura da superfície. Culturas com baixa cobertura vegetal, como as pastagens no norte da bacia e áreas de cana-de-açúcar, já colhidas, também estão relacionadas às temperaturas mais elevadas. As zonas mais quentes são as áreas urbanas, no entanto, pode haver uma inversão em condições de déficit hídrico elevado, como no ano de 1999 cujo déficit hídrico foi de 130mm. Em 1999 os remanescentes florestais, próximos as pastagens, na porção central da bacia, foram influenciados pela elevada temperatura do entorno. Apenas os maiores fragmentos de áreas vegetadas, principalmente plantios florestais e 

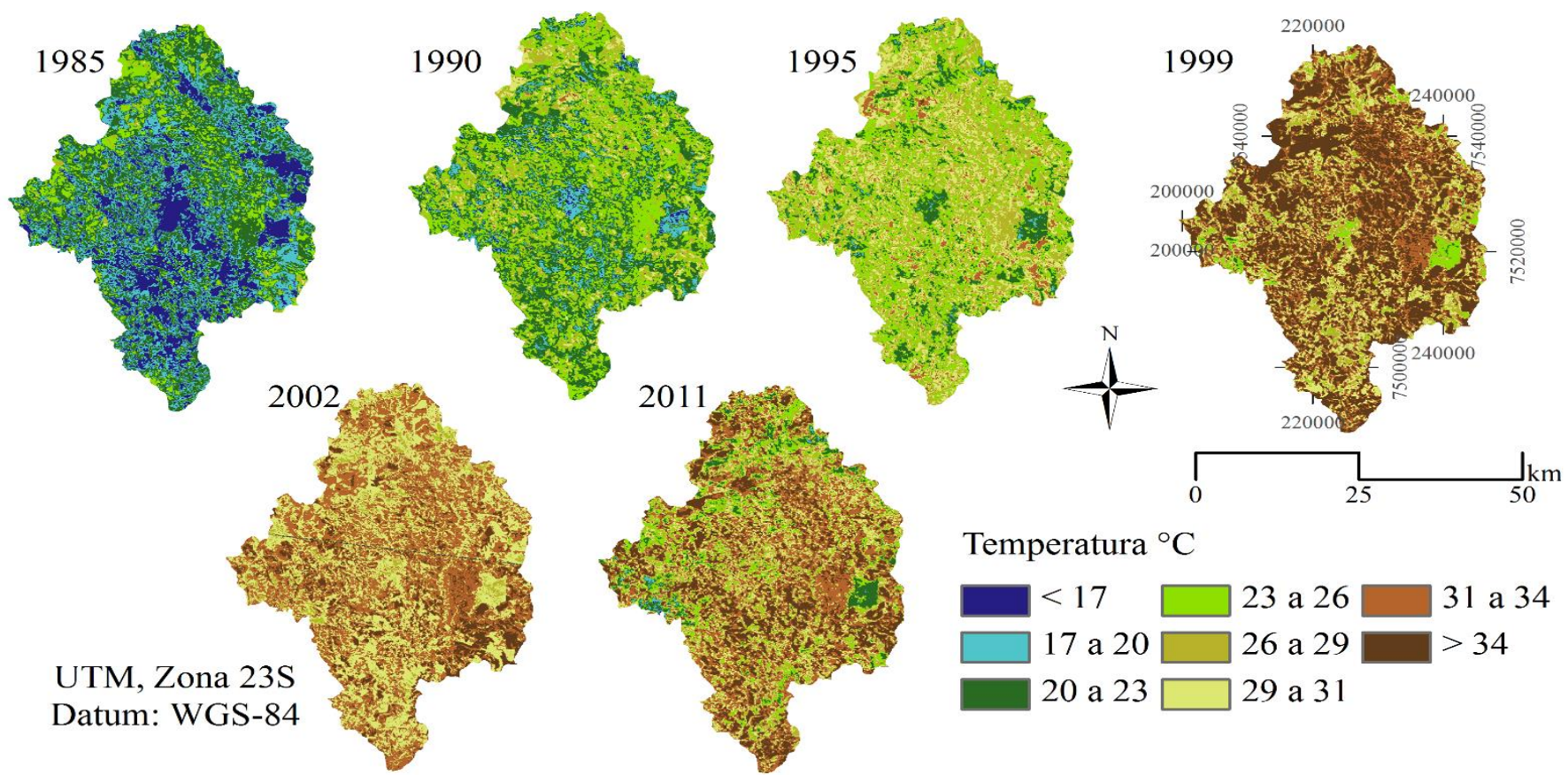

Temperatura ${ }^{\circ} \mathrm{C}$ Figura 4 - Variabilidade espacial da temperatura da superfície da bacia do Rio Corumbataí para os anos de
1985, 1990, 1995, 1999, 2002 e 2011.

A TSS foi negativamente correlacionada com os índices NDVI e MI e positivamente correlacionada com o NDBI, porém não houve correlação entre a altitude com nenhuma das outras variáveis (Figura 5). Orhan, Ekercin E DadaserCelik (2014) e Weng e Fu, (2014) realizaram estudo para verificar a variação da TSS e sua relação com as diferentes superfícies, respectivamente, na Turkia e Estados Unidos da América por meio dos índices NDVI e NDBI. De acordo com os autores, as maiores temperaturas foram observadas em zonas menos vegetadas, com baixos valores de NDVI e altos valores de NDBI.

Tais resultados indicam que as propriedades dos plantios florestais e os remanescentes propiciam condições para redução da TSS diferentes das outras classes, principalmente a pastagem, que são de baixa qualidade, e a cana-de-açúcar pela exposição do solo após a colheita. O comportamento observado pode estar relacionado à alteração de duas propriedades da superfície: a emissividade e o albedo.

A relação com a emissividade foi mostrada em estudos realizados por Hulley, Hook e Baldridge (2010) e French et al. (2008) que demonstraram que a TSS é função da emissividade da superfície. Foi observado que a emissividade do solo varia de 0,69 a 0,97, de acordo com o tipo de solo, teor de umidade e comprimento de onda. Em superfícies vegetadas, a emissividade varia ainda de acordo com o índice de área foliar, podendo ter valores acima de 0,99. Portanto, solo exposto e áreas urbanas têm maior capacidade de armazenar calor do que superfícies vegetadas, consequentemente, temperaturas na superfície mais elevadas (LIU et al., 2012).

Os resultados apresentados, corroboram com o comportamento observado na BRC, pois áreas de cana-deaçúcar colhida e pastagens com baixo índice de cobertura vegetal têm emissividade semelhante ao solo, ou seja, maior capacidade de retenção de calor causando um aumento relativo da temperatura. Os valores elevados de TSS na fruticultura são resultado da baixa emissividade que segundo Caselles e Sobrino (1989) varia de 0,947 a 0,984, dependendo da estrutura do pomar.

O albedo é a razão entre a energia eletromagnética refletida e incidente sobre um alvo. A umidade do solo, tipo de superfície, condições meteorológicas instantâneas e ângulo de elevação solar são os principais fatores determinantes do albedo em solo exposto (ZHANG et al., 2013; ZHANG et al., 2014). Em superfícies vegetadas o albedo é influenciado, dominantemente, pelo estádio de desenvolvimento da vegetação, pelo índice de área foliar e ângulo de inclinação das folhas (BSAIBES et al., 2009). Desta forma, o albedo da superfície e a umidade do solo são importantes fatores de controle da transferência de água e calor na interface superfície da terra-atmosfera, bem como efeito direto no balanço de radiação à superfície, principalmente sobre o fluxo de calor latente.

Na Figura 5, observou-se que a correlação entre a TSS, NDBI, NDVI e MI foi mais forte, podendo estar relacionado ao aumento das áreas de plantios florestais e remanescentes florestais, bem como a preservação dos 
remanescentes, fatores que favoreceram o aumento do NDVI. Por outro lado, as pastagens permaneceram sob sistema de manejo extensivo, provavelmente alcançando estádios mais avançados de degradação, e houve aumento das áreas de cana-de-açúcar, o que proporcionou maior área de solo exposto no período da colheita, fatores que promovem a redução do NDVI e aumento da resposta do NDBI. Tais condições afetaram a distribuição espacial e a magnitude da TSS.

O período de 2002 a 2011 foi o período em que houve um aumento expressivo do remanescente florestal e plantios florestais na BRC, sendo evidenciado na resposta do NDVI com média de 0,68 em 2002 e 0,94 em 2011. Tal período corresponde ao período de conversão do sistema de colheita da cana-de-açúcar no estado de São Paulo, que iniciou em 2002. A conversão do sistema de colheita da cana-de-açúcar pode ter contribuído com a recuperação dos remanescentes da vegetação ripária e o aumento da qualidade do remanescentes mais antigos, devido ao efeito de borda causado pelas queimadas. Pois, segundo Brando et al. (2014), práticas de uso agrícola, como a queima regular da palhada da cana-de-açúcar, têm influências negativas sobre $o$ fechamento e/ou manutenção das bordas. Ainda, segundo Nascimento e Laurance (2004) os efeitos de borda sobre os fragmentos florestais causam morte das árvores e consequente redução da biomassa dos fragmentos.
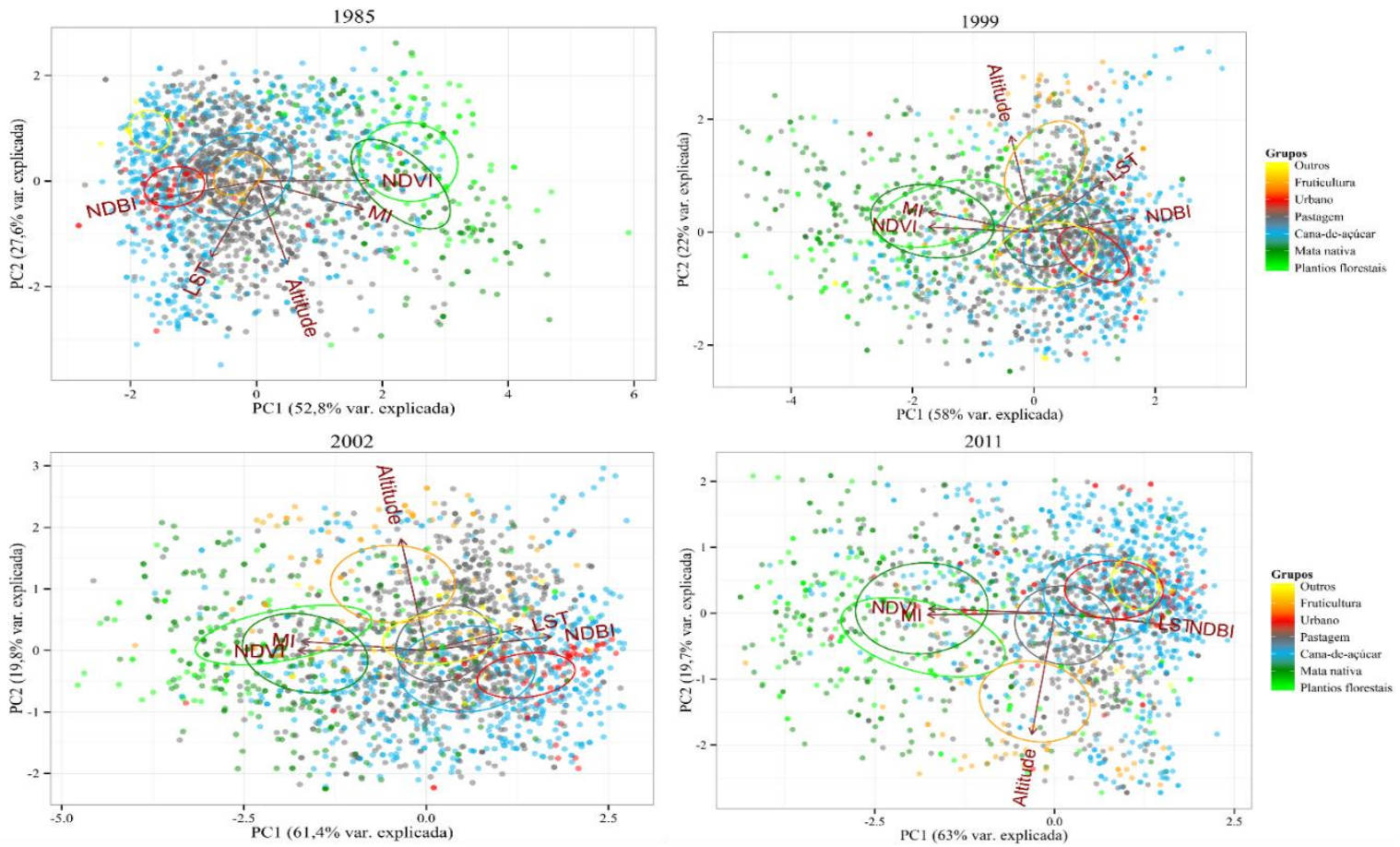

Figura 5 - Análise de componentes principais com as variáveis LST, NDVI, NDBI, MI e altitude da bacia do Rio Corumbataí, para os anos de 1985, 1999, 2002 e 2011.

O diagrama teórico do MI, é apresentado na forma de um triângulo retângulo, cuja abscissa representa a linha de vegetação, a ordenada a linha de água e a hipotenusa o gradiente de umidade. A origem deste diagrama, quando o MI é igual a zero, representa corpos d'água profundos (100\% de umidade e ausência de vegetação), consequentemente menor TSS; logo, o extremo oposto da linha de água representa a ausência de água (0\% de umidade) e maior TSS. O deslocamento à direita na linha de vegetação representa o aumento da cobertura vegetal, que corresponde ao maior valor de MI. Desta forma, o eixo do gradiente de umidade representa a relação entre a quantidade de cobertura vegetal e a umidade do ambiente. O ponto mais alto da linha do gradiente de umidade, adjacente à linha de água, representa os locais mais quentes e secos, enquanto que o extremo oposto desta linha representa os locais mais amenos e úmidos (Figura 6).

O período de agosto a setembro, na BRC, caracteriza-se por condições de déficit hídrico aliado a exposição do solo pela colheita da cana-de-açúcar, juntamente com a baixa qualidade das pastagens criam condições propícias para o aumento da TSS. Apenas as áreas com maior cobertura vegetal, plantios florestais, remanescentes e algumas áreas não colhidas de cana-de-açúcar, são capazes de manter a temperatura mais amena (Figura 6).

A maior dispersão dos pontos representados pela classe plantios florestais e remanescentes pode ser devido ao tamanho dos fragmentos de mata nativa, pois fragmentos pequenos perdem umidade para $\mathrm{o}$ ambiente $\mathrm{e}$, consequentemente, aumentam a sua temperatura. Quanto aos plantios florestais, pode ser devido à heterogeneidade dos plantios, ou seja, na época de obtenção da imagem havia talhões de diferentes idades. O padrão de distribuição de pontos no diagrama teórico do ano 2002 e 2011 também indicam a melhora da qualidade dos remanescentes florestais, com maior concentração de ponto na extremidade da linha de vegetação em 2011 em comparação com 2002. 


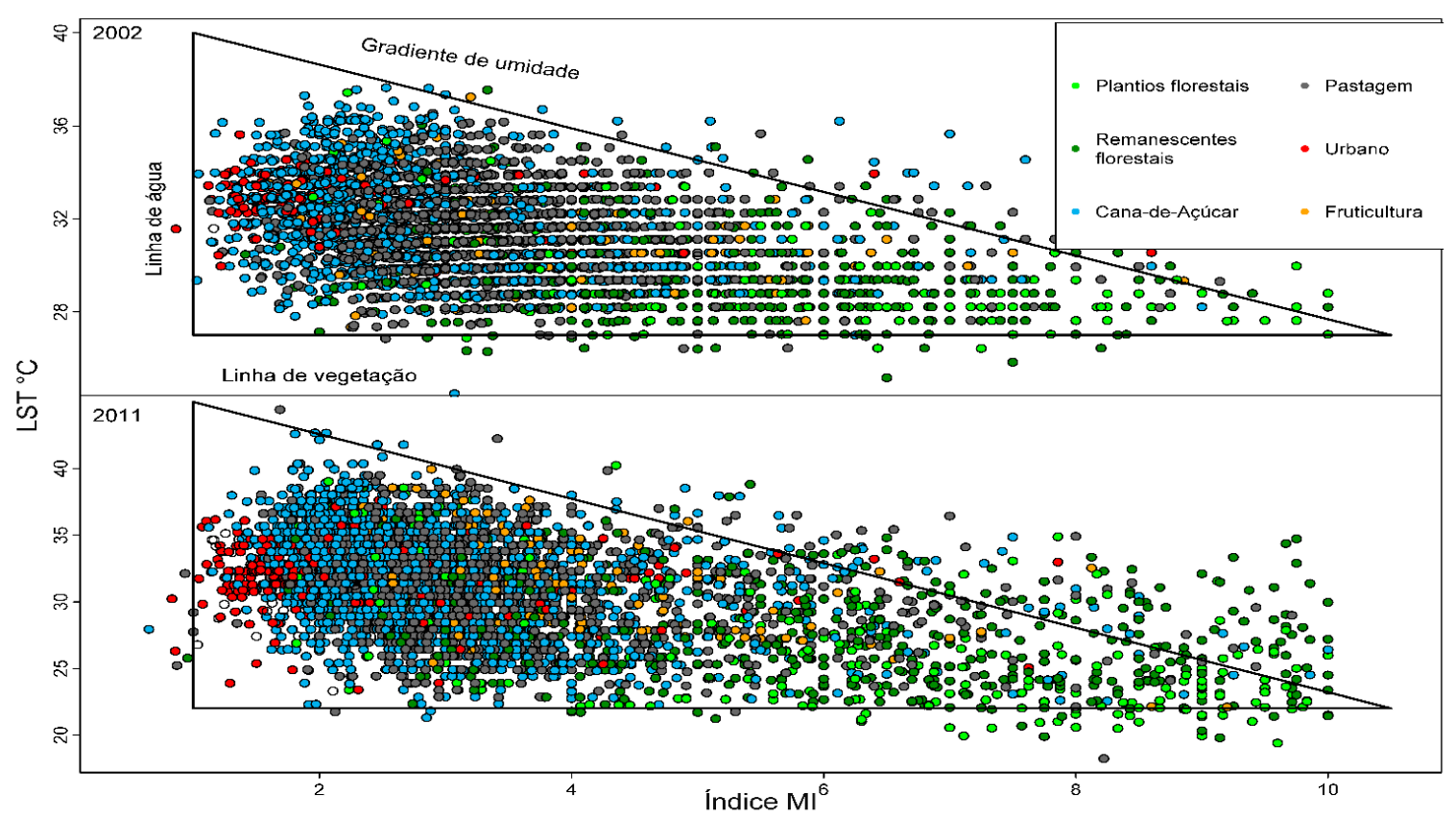

Figura 6 - Diagrama teórico do índice de umidade nos anos de 2002 e 2011.

\section{CONCLUSÕES}

$\checkmark \quad$ A variação espacial da temperatura da superfície do solo na bacia do Rio Corumbataí pode ser explicada pelo tipo de uso e cobertura do solo e mudanças no manejo das culturas pode, em algum nível, interferir positiva ou negativamente no padrão de distribuição térmica da bacia. Cobertura do solo que privilegiem a manutenção de cobertura vegetal e umidade do solo, como plantios florestais e recuperação florestal, tem potencial para redução da temperatura da superfície.

$\checkmark \quad$ A mudança no sistema de colheita da cana-deaçúcar pode ter provocado uma recuperação dos remanescentes florestais, consequentemente, as áreas com temperaturas mais amenas aumentaram.

\section{AGRADECIMENTOS}

À Escola Superior de Agricultura "Luiz de Queiroz" pela estrutura oferecida para a realização deste trabalho e ao $\mathrm{CNPq}$ pela concessão da bolsa e recursos para o desenvolvimento da pesquisa.

\section{REFERÊNCIAS}

BRANDO, P. M.; BALCH, J. K.; NEPSTAD, D. C.; MORTON, D. C.; PUTZ, F. E.; COE, M. T.; ALENCAR, A. Abrupt increases in Amazonian tree mortality due to drought-fire interactions. Proceedings of the National Academy of Sciences of the United States of America, Washington. v. 111, n. 17, p. 6347-6352, 2014.

BSAIBES, A.; COURAULT, D.; BARET, F.; WEISS, M.; OLIOSO, A.; JACOB, F.; HAGOLLE, O.; MARLOIE, O.;
BERTRAND, N.; DESFOND, V.; KZEMIPOUR, F. Albedo and LAI estimates from FORMOSAT-2 data for crop monitoring. Remote Sensing of Environment, Nova York. v. 113, n. 4, p. 716-729, 2009.

CASELLES, V.; SOBRINO, J. A. Determination of frosts in orange groves from NOAA-9 AVHRR DATA. Remote Sensing of Environment, Nova York. v. 29, n. 2, p. 135-146, 1989.

CHANDER, G.; MARKHAM, B. Revised Landsat-5 TM radiometric calibration procedures and postcalibration dynamic ranges. Geoscience and Remote Sensing, IEEE Transactions on, Caceres. v. 41, n. 11, p. 2674-2677, 2003.

CHANDER, G.; MARKHAM, B. L.; HELDER, D. L. Summary of current radiometric calibration coefficients for Landsat MSS, TM, ETM+, and EO-1 ALI sensors. Remote Sensing of Environment, Nova York. v. 113, n. 5, p. 893-903, 2009.

CHEN, T. DE JEU, R.; LIU, Y. Y.; VAN DER WERF, G. R.; DOLMAN, A. J. Using satellite based soil moisture to quantify the water driven variability in NDVI: A case study over mainland Australia. Remote Sensing of Environment, Nova York. v. 140, p. 330-338, 2014.

DUPIGNY-GIROUX, L.-A.; LEWIS, J. E. A moisture index for surface characterization over a semiarid area. Photogrammetric Engineering and Remote Sensing, Nova York. v. 65, p. 937-946, 1999.

FRENCH, A. N.; SCHMUGGE, T. J.; RITCHIE, J. C.; HSU, A.; JACOB, F.; OGAWA, K. Detecting land cover change at the Jornada Experimental Range, New Mexico with ASTER emissivities. Remote Sensing of 
Environment, Nova York. v. 112, n. 4, p. 1730-1748, 2008.

GRODZKI, L.; CARAMORI, P. H.; BOOTSMA, A.; OLIVEIRA, D. D.; GOMES, J. Riscos de ocorrência de geada no Estado do Paraná. Revista Brasileira de Agrometeorologia, Santa Maria, v. 4, n. 1, p. 93-99, 1996.

HULLEY, G. C.; HOOK, S. J.; BALDRIDGE, A. M. Investigating the effects of soil moisture on thermal infrared land surface temperature and emissivity using satellite retrievals and laboratory measurements. Remote Sensing of Environment, Nova York. v. 114, n. 7, p. 1480-1493, 2010.

LIU, Y.; SHINTARO, G.; ZHUANG, D.; KUANG, W. Urban surface heat fluxes infrared remote sensing inversion and their relationship with land use types. Journal of Geographical Sciences, Berlim. v. 22, n. 4, p. 699-715, 2012.

NASCIMENTO, H. E. M.; LAURANCE, W. F. Biomass dynamics in Amazonian forest fragments. Ecological Applications, Nova York. v. 14, n. 4, p. S127-S138, 2004.

ORHAN, O.; EKERCIN, S.; DADASER-CELIK, F. Use of Landsat Land Surface Temperature and Vegetation Indices for Monitoring Drought in the Salt Lake Basin Area, Turkey. Scientific World Journal,Nova York. p. 11, 2014.

QIN, Z.; KARNIELI, A.; BERLINER, P. A monowindow algorithm for retrieving land surface temperature from Landsat TM data and its application to the IsraelEgypt border region. International Journal of Remote Sensing, Londres. v. 22, n. 18, p. 3719-3746, 2001.

STOLL, M. J.; BRAZEL, A. J. Surface-air temperature relationships in the urban-environment of Phoenix, Arizona. Physical Geography, Abingdon. v. 13, n. 2, p. 160-179, Apr-Jun 1992. ISSN 0272-3646.

R DEVELOPMENT CORE TEAM (2008). R: A language and environment for statistical Computing. $R$ Foundation for Statistical Computing, Vienna, Austria. ISBN 3-900051-07-0, URL http://www.R-project.org.

TROPPMAIR, H. Variação da estrutura da mata galeria na bacia do rio Corumbataí (SP) em relação à água do solo, do tipo de margem e do traçado do rio. São Paulo: USP-Instituto de Geografia, 1974.

VALENTE, R. D. O. A.; VETTORAZZI, C. A. Definition of priority areas for forest conservation through the ordered weighted averaging method. Forest Ecology and Management, Amsterdam. v. 256, n. 6, p. 1408-1417, 2008.

VALENTE, R. O. A.; VETTORAZZI, C. A. Análise da estrutura florestal na Baica do Rio Corumbataí, SP. Scientia Forestalis, Piracicaba. n.62 p. 15, 2002.

VALERIANO, M.M.; KUPLICH, T. M.; STORINO, M.; AMARAL, B. D.; MENDES, J. N.; LIMA, D. J.
Modeling small watersheds in Brazilian Amazonia with shuttle radar topographic mission-90 $\mathrm{m}$ data. Computers \& Geosciences, Amsterdam. v. 32, n. 8, 2006.

VAN DE GRIEND, A. A.; OWE, M. On the relationship between thermal emissivity and the normalized difference vegetation index for natural surfaces. International Journal of Remote Sensing, Londres. v. 14, n. 6, p. 1119-1131, 1993.

WENG, Q. H.; FU, P. Modeling annual parameters of clear-sky land surface temperature variations and evaluating the impact of cloud cover using time series of Landsat TIR data. Remote Sensing of Environment, Nova York. v. 140, p. 267-278, 2014.

ZHAN, W.; CHEN, Y.; VOOGT, J.; ZHOU, J.; WANG, J.; LIU, W.; MA, W. Interpolating diurnal surface temperatures of an urban facet using sporadic thermal observations. Building and Environment, Amsterdam. v. 57, p. 239-252, 2012.

ZHANG, Y.; ODEH, I. O. A.; HAN, C. Bi-temporal characterization of land surface temperature in relation to impervious surface area, NDVI and NDBI, using a sub-pixel image analysis. International Journal of Applied Earth Observation and Geoinformation. Amsterdam. v. 11, n. 4, p. 256-264, 2009.

ZHANG, Y. F.; WANG, X. P.; HU, R.; PAN, Y. X.; ZHANG, $H$. Variation of albedo to soil moisture for sand dunes and biological soil crusts in arid desert ecosystems. Environmental Earth Sciences, Londres. v. 71, n. 3, p. 1281-1288, 2014.

ZHANG, Y. F.; WANG, X. P.; PAN, Y. X.; HU, $R$. Diurnal and seasonal variations of surface albedo in a spring wheat field of arid lands of Northwestern China. International Journal of Biometeorology, Nova York. v. 57, n. 1 , p. $67-73,2013$. 